Théologiques

Théologiques

\title{
Dieu, don, animal et la question du sujet
}

\section{Marc De Kesel}

Volume 22, numéro 1, 2014

URI : https://id.erudit.org/iderudit/1033099ar

DOI : https://doi.org/10.7202/1033099ar

Aller au sommaire du numéro

\section{Éditeur(s)}

Faculté de théologie et de sciences des religions, Université de Montréal

\section{ISSN}

1188-7109 (imprimé)

1492-1413 (numérique)

Découvrir la revue

\section{Citer cet article}

De Kesel, M. (2014). Dieu, don, animal et la question du sujet. Théologiques, 22(1), 143-159. https://doi.org/10.7202/1033099ar

\section{Résumé de l'article}

Les tentatives contemporaines de donner une place sociale et politique à l'animal et de lui attribuer le statut de « sujet " renvoie toujours aussi à la question concernant le sujet humain, plus spécifiquement le sujet de la modernité. C'est parce que nous sommes trop sûrs (c'est-à-dire pas du tout sûrs) concernant ce qu'est le " sujet moderne ", que nous inventons la question sur l'animalité. D’une certaine manière, pour le problème du sujet de la modernité, la question de l'animal a une fonction similaire à la question de Dieu. C'est pourquoi une référence à la tradition théologique chrétienne est nécessaire pour comprendre l'enjeu de notre fascination actuelle pour l'animalité. Analysé à partir du paradigme du don (conçu par Marcel Mauss), l'essai offre une courte généalogie de la relation de l'homme à l'animal et montre comment la religion (dans toutes ses formes) y est profondément impliqué. 


\title{
Dieu, don, animal et la question du sujet
}

\author{
Marc De KeseL* \\ Théologie \\ Université Saint-Paul, Ottawa (Canada)
}

\section{Le don de l'animal}

Encore au XIX ${ }^{\mathrm{e}}$ siècle, les peuples chamanistes de Sibérie vivaient de la chasse et, par conséquent, tuaient des animaux. Mais ils ne le faisaient jamais sans rituels complexes. Prétendre qu'on comprend tout-à-fait ce que de tels phénomènes veulent dire n'est jamais sans risque, mais à croire les anthropologues, quelques traits dans les «danses» de ces chamanistes sont pourtant assez clairs: ces « rituels»

constituent en fait une sorte de chasse symbolique dirigée et mimée dans les danses que fait le chamane avec différents masques d'animaux. Plus encore, cette chasse symbolique est conçue comme une union matrimoniale entre l'époux chasseur et la bête chassée; le chamane mime le mâle en rut qui cherche à séduire son épouse animale. Tout le rite se déroule autour de ce motif de l'alliance qui, outre l'union avec la bête, inclut celle avec les esprits de la forêt et avec les autres animaux. Toutes ces relations sont conçues comme des relations de partenaires qui à la fois se respectent, se provoquent, s'attirent et se réjouissent dans leurs échanges, c'est pourquoi «les jeux plaisent aux esprits ». (Hénaff 2002, 184-185; l'auteur cite Hamayon 1995, 80)

Les Sibériens considéraient les animaux comme des jeunes femmes à courtiser, mais cela ne les gênait nullement d'aller les tuer pendant la chasse. Les hommes étaient bien conscients qu'ils vivaient des animaux, ils

Marc De Kesel est professeur agrégé en philosophie à l'Université Saint-Paul (Ottawa, Canada). Ses champs de recherches sont l'éthique publique, la philosophie continentale, la philosophie politique, la relation entre théologie et philosophie, les études des religions, les études sur la Shoah et la théorie freudo-lacanienne. Entre autres, il a récemment publié (2009) Eros \& Ethics, Reading Jacques Lacan's Seminar VII, Albany, SUNY et (2012) Auschwitz mon amour, Amsterdam, Boom. 
leur étaient manifestement reconnaissants pour ça, tout en les tuant et les mangeant.

Phénomène assez paradoxal, sauf si on y reconnait une logique de don. Il est un fait que, aux yeux de ces Sibériens (comme pour tous les peuples dits «archaïques»), tout ce qui existe est donné: la vie des hommes et celle des animaux aussi bien que la mort et la capacité humaine de tuer ces animaux. Et c'est en reconnaissant cette condition générale du don que l'homme "archaïque " légitime la chasse. Les animaux se donnent à l'homme, et la chasse n'est qu'une manière pour «recevoir » et «accepter » leur don - tout comme la danse rituelle qui précède la chasse est un "contredon» de la part des hommes exprimant leur respect envers la grâce qui leur est rendue. Bref, la chasse est un jeu composé «de donner, de recevoir, et de rendre ", les trois composantes du «fait social total » qu'est le don, comme le formulait le sociologue et anthropologue Marcel Mauss dans son fameux Essai sur le don (2012, 82-85).

Les Kwakiutl, Tinglit, Haïda, et tant d'autres peuples indigènes de la côte nord-ouest de l'Amérique (pour nous limiter à un seul autre exemple ethnologique), exprimaient le fait qu'ils vivaient de l'abondance des poissons qui leur étaient offerts par les mers et les rivières (Drucker 1965, 94-95; voir aussi Hyde 2007, 33-34). Un de leurs mythes racontait que les saumons étaient en fait la métamorphose d'un peuple humain qui vivait dans les profondeurs de la mer. Pour donner aux hommes de la terre une abondante nourriture, ces "frères" étaient si gentils qu'ils changeaient leur corps en celui d'un saumon, quittaient leur monde maritime et, nageant en amont, entraient dans les rivières afin de se donner aux hommes. C'est pourquoi ceux-ci accueillaient solennellement le premier saumon, le mangeaient précautionneusement sans casser ses arêtes pour que l'on puisse rendre celles-ci à la rivière où le saumon reprendrait son corps de poisson puis, une fois revenu au sein de la mer, son corps humain. Ainsi le saumon garantissait le cycle de la vie et de la mort, un cycle qu'on considérait comme géré par le paradigme du don.

Pour les théoriciens du don, c'est à partir de cette "prestation sociale totale» du don que se développaient les religions dans l'histoire (Mauss 2012, 73-75; pour une élaboration exhaustive de ce point de vue, voir Tarot 2008). Progressivement, les agents du don, d'abord identifiés aux hommes eux-mêmes, se changeaient en «ancêtres », puis en "esprits » et en "dieux». C'est la raison pour laquelle beaucoup de cultures — comme celle de l'Égypte Ancienne - ont un panthéon rempli de dieux animaux ou partiellement animaux. C'est parce que l'animal donne la vie à l'homme, 
qu'il est divin, de la même façon que c'est le caractère divin de la vie qui rend le dieu animal. Dans ces cultures, l'animal et le divin indiquaient le côté «don» dans «l'économie» de laquelle vivait l'homme.

\section{L'animal et le don «sujet»}

On pourrait dire que dans les temps anciens, l'animal remplissait le rôle de «sujet ». Non pas qu'il était l'égal de l'homme, loin de là. L'animal était considéré comme lié aux dieux. Si l'animal était "sujet", ceci est à comprendre dans le sens strict du terme, c'est-à-dire dans le sens qu'il appartenait à ce qui est «jeté sous» - et par conséquent formait «la base de» — la manière à laquelle l'homme se rapporte au monde. "Subjectum", «sujet» est le terme pour le support, le fondement sur lequel l'homme se "pose» en se référant au monde. Le terme indique la supposition fondamentale à partir de laquelle il construit son monde, y compris lui-même. En fait, «sujet» est lexicalement similaire au mot «supposé » : ce qui est «posé sous » : notre fond ou support, le point fondamental à partir duquel nous nous rapportons à la réalité (voir Balibar, Cassin et de Libera 2004).

Dans ces temps-là, en effet, l'homme lui-même ne se considérait pas comme "sujet». Il ne se définissait pas comme le point à partir duquel il se référait au monde. Il ne considérait pas sa relation au monde comme partant de lui-même - d'un soi considéré comme fondé en soi. Il ne se comprenait pas comme un soi autonome, mais comme donné. Même son sentiment de "soi ", était vécu comme primordialement donné — donné comme tout l'était dans l'univers. Ce n'était pas lui qui était sujet, c'était l'agent de ce don universel — réalisé entre autres dans l'animal qui s'offrait à lui dans la chasse.

Mais ceci veut en même temps dire qu'il n'y avait pas un sujet, un sujet un, uni ou unifié. Tout était donné, mais non par une instance une - l'animal, la communauté des dieux ou le dieu unique. Tout était donné dans le sens où tout était immergé dans un trafic de don-réception-contredon. Tout était donné à tout ce qui est, parce que tous donnaient à tous. Et tous étaient liés parce qu'ils ne s'arrêtaient pas de se donner des "présents" les uns aux autres. C'est d'ailleurs aussi ce trafic qui donnait à chacun des trafiquants un «soi », une identité. C'est pour cette raison que les Kwakiutl de la côte ouest de l'Amérique du Nord tenaient les saumons pour humains. Le «sujet» - le point à partir duquel l'homme se comprenait lui-même - était l'échange mutuel des dons en tant que tel. Finalement, c'est cet échange qui était leur sujet. 
On comprend mieux maintenant pourquoi les hommes archaïques ne voyaient pas d'opposition entre respecter et tuer un animal. Chez les Aïnous japonais, chez les Jankuntjara australiens ou les Gogodala de la Nouvelle Guinée, on n'était pas surpris de voir une femme donner son sein à un animal, qu'ensuite on gardait pour nourrir et soigner, et qu'on finissait par tuer et manger (Milliet 1987; 2003). Cela se laisse comprendre à partir du cercle du don, un cercle qui n'a nullement son origine dans un au-delà transcendent, mais qui n'existe que dans l'échange mutuel concret des dons et des contredons.

C'est précisément ce qui changera au cours de l'histoire. Ce n'est pas dire qu'on perdra l'idée que tout est donné. On pourrait même dire qu'elle deviendra de plus en plus forte et rencontrera son acmé dans les religions monothéistes. Mais le changement décisif consiste dans le fait qu'on substantivera le don. On lui attribuera un statut de plus en plus indépendant de sorte que tout sera donné à partir d'une instance exclusivement donnante. C'est ainsi qu'on peut interpréter l'émergence des religions dans l'histoire. Le don n'y est plus considéré comme ayant son départ chez les hommes qui donnent l'un à l'autre tout ce que «est », y compris «euxmêmes "; le don religieux, en revanche, part d'un domaine donateur relativement autonome: le domaine des ancêtres ou celui des dieux.

Néanmoins, ces dieux n'étaient pas eux-mêmes indépendants des dons que leur adressaient les humains. Si les mortels ne pouvaient pas vivre sans les dons des immortels, ces derniers ne pouvaient vivre non plus sans les dons des hommes. Ce n'était que par des dons - des sacrifices, des prières, des processions, des temples, des rites, etc. - que les dieux pouvaient être ce qu'ils étaient et tenir leur renommée - leur «fama» — qui était la garantie de leur «immortalité». Sans les contredons des hommes, les dieux cessaient d'être la source des dons nourrissant la vie humaine. Entre les hommes et les dieux, il y avait un trafic des dons et c'était finalement ce trafic qui donnait la vie, aussi bien aux dieux qu'aux hommes.

Vivant des animaux, l'homme païn vénérait ce don en rendant à ses dieux la figure d'un animal — ou, peut-être plus exactement, en rendant à son animal la figure d'un dieu. Comme déjà suggéré, ce n'est pas un hasard si les panthéons des religions archaïques et antiques sont remplis d'animaux ou de références à l'animal.

Si on suit la suggestion faite par Jean-Pierre Vernant et Marcel Détienne (1972), on pourrait dire que manger de la viande est l'acte religieux par 
excellence ${ }^{1}$. Car qu'est-ce qu'un sacrifice, sinon une "cuisine» où, il est vrai, on offre quelques pièces de l'animal brulé aux dieux, mais où la plupart de la bête rôtie est servie à la communauté réunie pour le banquet de fête. Le don adressé aux dieux n'est pas séparé du don fait par des dieux - un don qui se manifeste aussi dans le donner à manger à la cité réunie (voir aussi Fontenay 1998, 217-225).

On tue des animaux, non parce qu'ils sont considérés comme inférieurs à l'homme. Au contraire, on les tue précisément parce qu'ils sont supérieurs - car sacrés. C'est d'eux que part le don dont vit l'homme et c'est leur don qui constitue le support — le «sujet» — des dons des hommes entre eux. La grammaire religieuse du don nous fait comprendre que tuer un animal implique une reconnaissance de son statut sacré et une stimulation du trafic des dons qui constitue la société humaine.

\section{L'animal et le donneur «sujet»}

C'est dans cette grammaire religieuse du don que le monothéisme introduira une rupture qui, étant donné son succès, sera décisive pour la suite de l'histoire de l'Occident - et par conséquent de celle du monde entier. Cette rupture changera le rapport de l'homme à l'animal, annonçant le rapport moderne à ce dernier.

Cette rupture est l'effet d'un nouveau critère que le monothéisme met en place dans le domaine du religieux: celui de la vérité. Pour cette tradition, la relation humaine envers le divin doit passer par le critère distinguant un dieu vrai d'un dieu faux. Car seul le dieu vrai est digne de culte. Et, comme la vérité ne peut pas être plurielle, le dieu monothéiste ne peut l'être non plus. Comme la vérité, il ne peut être qu'un et indivisible. Il n'y a pas des vrais dieux, il ne peut y avoir qu'un seul dieu qui soit vrai - ou, ce qui est dire la même chose: ce n'est que la vérité une, inébranlable et invariable qui est Dieu².

Tel est le Dieu introduit pour la première fois dans l'histoire par la «révolution religieuse» lancée par le pharaon égyptien Akhenaton au XIV $^{\text {ème }}$ siècle avant J.C. - une révolution qui d'ailleurs est restée sans succès. Ce n'est que l'esprit religieux réformiste des Hébreux qui, dans la première

1. Voir, par exemple, p. 67: «L'alimentation carnée coïncide absolument avec la pratique sacrificielle; toute viande consacrée est une victime animale égorgée rituellement, et le boucher qui fait couler le sang des bêtes porte le même nom fonctionnel que le sacrificateur posté près de l'autel ensanglanté.»

2. Pour le monothéisme comme défini par le critère de la vérité, voir Assmann (2007). 
moitié du premier millénaire avant J.C., a mis en place une culture monothéiste durable. Installée au sein d'un seul petit peuple, cette religiosité monothéiste est devenue dominante à travers les traditions chrétiennes et islamiques. Parlant de religion aujourd'hui, on se réfère à la grammaire de la religiosité monothéiste, les incroyants aussi bien que les croyants.

Cette religiosité monothéiste diffère profondément de la "grammaire » sacrale des religions dite païennes. Ici, la perspective du don pourrait être éclairante. La religiosité monothéiste, elle aussi, est sensible à la dimension du don qui marque tout ce qui est et qui est constitutive de la sociabilité de l'homme. Pour elle aussi, tout ce qui existe est le résultat d'un don. Mais elle modifie profondément le sujet de ce don. Selon la sensibilité des religions non-monothéistes, le sujet du don qui fonde la vie était le trafic des dons, un trafic bilatéral qui va des dieux vers les hommes aussi bien que des hommes vers les dieux.

Ceci ne vaut plus pour le Dieu monothéiste. Celui-ci donne, mais il donne sans être lui-même dépendant des dons des autres: ni des autres dieux (étant le vrai dieu, il est le seul), ni des hommes. C'est précisément cela qui signifie qu'il est vrai, qu'il coïncide avec la vérité: son existence ne dépend que de lui-même, il "est ce qu'il est " (comme on peut traduire le "Eyeh asher eyeh» d'Exode 3,14), indépendant de sa fama, de sa renommée parmi les hommes. Il est éternel, non dans le sens archaïque ou mythique du terme (c'est-à-dire vivant dans la mémoire des générations futures), mais dans le sens philosophique et scientifique: éternel, même si personne ne parle de lui, même si la majorité des hommes dénie son éternité et sa vérité. Sa vérité est précisément ce qui le place au-delà des opinions humaines.

Le dieu monothéiste donne tout et, comme son existence, son don aussi est indépendant des contredons de la part des hommes. Le monothéisme implique une rupture avec la religion définie comme commerce entre mortels et immortels, c'est-à-dire comme «trafic des dons ». Nous ne vivons que des dons prêtés par Dieu, mais Dieu lui-même ne vit pas de nos dons. Il n'a pas besoin des sacrifices, bien qu'il les tolère: dans un lieu seulement, son temple unique à Jérusalem, et non sans préciser que c'est la Loi (la Torah), plutôt que les sacrifices, qui définit le mieux le rapport à Lui. S'il y a don, c'est entre les hommes, c'est le don donné "aux veuves et aux orphelins ", comme le dit la formule biblique (notamment Ex 22,20; Dt 10,17-18; Is 1,17). Mais les dons que les hommes se donnent ne font sens que dans la mesure où ils participent au don unilatéral venant de leur Dieu unique. C'est Dieu qui donne tout sans lui-même vivre des dons, et si 
les hommes donnent entre eux, c'est parce que la Loi leur ordonne de le faire. Ce n'est plus le don mutuel, le trafic des dons, qui est le «sujet», c'est le Dieu Unique Donneur.

Et où sont restées les bêtes? Comment les monothéistes traitent-ils les animaux? Quoiqu'objets de sacrifice, ceux-ci perdent leur statut sacré, car le sacrifice perd son efficacité sacrale, c'est-à-dire sa capacité d'entretenir une relation bilatérale avec le divin - relation précisément constitutive du divin païen. Le sacrifice n'effectue rien car il n'est devenu qu'un simple signe - signe qui, paradoxalement, symbolise aussi que Dieu n'a pas besoin de sacrifices. C'est pourquoi, quand le seul lieu sacrificiel, le temple de Jérusalem, disparait en l'an 70, le monothéisme juif peut continuer, tout comme le christianisme et l'islam ont pu sortir du monothéisme juif sans donner une place cruciale au sacrifice des animaux. Les sacrifices ne restent que des "signes ", signes qui distinguent par exemple les animaux considérés comme purs ou impurs - signes de la vérité qui règne sur le monde (voir, par exemple, Houston 1993). Ce n'est pas tant parce qu'il sacrifie des animaux que parce qu'il obéit à cette distinction entre "vrais » et "faux " animaux, que l'homme monothéiste se montre loyal au don donné par son Dieu unique.

\section{L'animal et l'homme «sujet»}

On pourrait dire que le monothéisme «capitalise» le don. Il le fixe exclusivement dans une seule instance donnante qui, elle, se soustrait au don. C'est pourquoi cette religiosité a fait de l'homme un «sujet» dans le seul sens d'assujetti - assujetti au don absolu d'un Dieu qui, lui-même, est l'unique "sujet» du don dans le sens d'agent, d'instance donnante du don.

On pourrait interpréter le mouvement émancipatoire qui est à la base de la modernité comme la tentative de se libérer de cette position assujettie et de devenir l'agent - le sujet actif — du don qu'est la vie. Cette aspiration à la liberté émerge dans la «révolution bourgeoise» qui accompagne la renaissance des villes en Europe Occidentale au XIII ${ }^{\mathrm{e}}$ siècle et ne fait que s'agrandir dans les siècles qui suivent. Mais en voulant être le sujet-agent du don qu'est la vie, l'homme évoluera vers une définition de soi comme libéré $d u$ don en tant que tel. En ce sens, la rupture entre le Moyen Âge chrétien et la Modernité est difficile à surestimer. Cette rupture réalise un véritable changement de paradigme. L'homme ne se comprend plus primordialement comme donné mais comme donnant, comme celui qui se donne lui-même la vie comme il le veut. Ce n'est pas dire qu'il nie le fait 
qu'il a été créé - et par conséquent "donné» — par Dieu, mais il ne se confie pas à cette idée à partir de sa condition "donnée ", mais à partir de sa condition libre. Il choisit librement sa croyance en Dieu. Et à la base de ce libre arbitre, le croyant peut respecter ceux qui font l'autre choix.

Voici le sujet moderne, comme il a été formulé pour la première fois de manière profondément réfléchie par René Descartes. On peut douter de tout ce qui est, ou comme Descartes disait plus exactement, on doit en douter, car c'est le seul chemin qui mène à ce qui est vraiment certain. Cette chose hors du doute se découvrira dans le douteur même. C'est précisément par un doute radical et hyperbolique que l'homme peut se découvrir soi-même comme y échappant, en tant que point de certitude, en tant que «sujet ». Même si, ontologiquement, il sait fort bien qu'il est donné/créé, il se trouve lui-même comme "sujet » dans le doute — le doute met tout en doute, même cela. Le doute méthodique de Descartes rompt avec le «sujet » comme l'avait conçu la science médiévale, c'est-à-dire le sujet défini comme don divin. Après Descartes, le point de départ qui fonde et organise notre relation au monde n'est plus le fait que l'homme et le monde sont donnés l'un à l'autre par le même donateur. Désormais, l'homme approche le monde à partir de lui-même, de son autonomie vis-à-vis du monde. Avec la modernité, c'est la liberté humaine qui est devenue "sujet».

Il est clair que cette liberté suppose une rupture radicale entre l'homme et le monde. Car c'est ce que la radicalité de la liberté moderne implique: d'être indépendant du monde autour de soi. Auparavant, l'homme se pensait participer à l'être autour de lui, ce qui est la raison pour laquelle il prétendait connaître l'essence des choses. Le christianisme a ajouté que cette essence restait au fond mystérieuse car elle était marquée par une origine divine qui échappe à la connaissance humaine. Néanmoins, la relation (intellectuelle et autre) aux choses concernait leur essence, une essence qu'on croyait créée, donnée, par Dieu. La relation moderne aux choses ne prétendait plus être ancrée dans l'essence. L'homme moderne n'a accès qu'à la surface des choses - un accès qui peut (et doit) se limiter à l'observation empirique. La physique arrête d'être essentialiste et devient exclusivement empirique: voilà la physique newtonienne qui engendrera le paradigme général de la science moderne. La modernité changera le monde en un univers mécaniste, un univers constitué des choses qui ne seront plus approchées en tant qu'elles ont une «vie » à l'intérieur d'elles (comme des corps vivants, par exemple), mais selon leur caractéristiques exclusivement extérieures (hauteur, largeur et profondeur), comme corps mathématiques. La modernité changera l'univers en un ensemble infini de choses mortes: 
un univers qui est radicalement différent de celui de la pensée elle-même qui est l'univers de la conscience, du vouloir, de la liberté, des sentiments, bref, l'univers de l'âme.

Voilà le dualisme cartésien qui deviendra le paradigme conduisant la relation de l'homme moderne au monde - un paradigme, il est vrai, problématique et problématisé tout au long de la modernité, mais qui est resté néanmoins dominant jusqu'à nos jours. C'est ce paradigme qui est à la base de la technique moderne: c'est parce que nous considérons les choses hors de nous comme libérées de toute essence, que nous pouvons faire avec eux ce que nous voulons. Elles sont radicalement réduites au statut d'objet; la fonction de sujet est intégralement du côté de l'homme qui, lui aussi, est libéré de tout essence mais qui est défini comme «agent» censé capable de traiter tout de qui est comme "objet», de par sa capacité technique libre et infinie.

Le premier «objet» à propos duquel le caractère problématique de ce dualisme typiquement moderne se manifeste, est l'animal. Pour l'antiquité aristotélicienne aussi bien que pour la scolastique médiévale imprégnée pas l'aristotélisme, l'homme et l'animal appartenaient au même «être»: l'homme était considéré comme un animal se distinguant de lui par quelques traits supplémentaires. Le paradigme cartésien apporte entre l'homme et l'animal une rupture radicale et jamais vue dans l'histoire: comme tout ce qui n'appartient pas au domaine libre du sujet, l'animal est considéré comme un "objet", comme un être déterminé qui fonctionne de manière entièrement mécanique. La vie n'est pas l'effet d'un principe de vie intérieure (comme c'était le cas dans la physique aristotélicienne "essentialiste»), mais l'effet d'une causalité radicalement extérieure. C'est-à-dire que les animaux sont vus comme des machines - des «automata» — qui répondaient à une logique strictement déterministe ${ }^{3}$. Tout comme le corps humain est vu comme une machine, séparée de cet autre «être» qu'il est, son âme, régie par l'auto-détermination, c'est-à-dire par la libertét

3. «Ce qui ne semblera nullement étrange à ceux qui, sachant combien de divers automates, ou machines mouvantes, l'industrie des hommes peut faire, sans y employer que fort peu de pièces, à comparaison de la grande multitude des os, des muscles, des nerfs, des artères, des veines, et de toutes les autres parties qui sont dans le corps de chaque animal, considéreront ce corps comme une machine, qui, ayant été faite des mains de Dieu, est incomparablement mieux ordonnée, et a en soi des mouvements plus admirables, qu'aucune de celles qui peuvent être inventées par les hommes " (Descartes 1969 ,69; voir aussi Clarke 2006, 333; Fontenay 1998, 275-288).

4. Pour le matérialisme du XvIII ${ }^{\mathrm{e}}$ siècle, l'homme ne sera que cette «machine». Voir par exemple le pamphlet philosophique de Julien Offray de La Mettrie, L'homme machine, de 1748 (La Mettrie 1981). 
Il y a cette anecdote qui raconte que Descartes, résidant à Alkmaar (aux Pays Bas), était sollicité par un visiteur pour visiter sa bibliothèque. Il le mena à la cour intérieure de sa maison où il était en train de faire une dissection sur une vache morte. "Voici, disait-il, ma bibliothèque " (Descartes 1901, 248). N'ayant plus de confiance dans le monde des livres, Descartes se livrait au "livre du monde», mais lisait cette métaphore médiévale d'une manière moderne: comme l'univers mécanique auquel appartenaient aussi tous les êtres dits "vivants».

Ce n'est qu'après deux siècles d'approche mécaniciste des animaux que, pour les sciences, les phénomènes de vie obtiennent un statut spécifique. L'émergence de la biologie moderne ne date que du début du XIX ${ }^{\mathrm{e}}$ siècle, commençant avec François-Xavier Bichat et n'établissant son paradigme de manière définitive qu'avec l'œuvre de Charles Darwin (voir Bichat 2013). Selon ce paradigme, la vie ne fonctionne pas de manière mécanique, elle n'est pas un système hydraulique complexe comme le pensait Descartes ni une «horloge » infiniment subtile comme on pensait encore au XVIII ${ }^{\mathrm{e}}$ siècle. La vie fonctionne à partir du principe "stimulus / réaction ", qui est un principe extérieur lui-aussi (contraire au principe intérieur qui domine la biologie aristotélicienne), tout en donnant aux phénomènes de vie un statut spécifique dans la totalité des objets de science.

Cependant, ceci ne concerne pas le «sujet» de notre rapport aux animaux. Le point à partir duquel nous approchons les phénomènes de vie reste structurellement localisé hors de ce domaine, hors de cette vie. Ce point de vue nous garantit une liberté vis-à-vis des animaux et nous place dans une position où en principe nous pouvons faire avec eux ce que nous voulons. Ils restent des objets d'une technique quasi-omnipotente et constamment tentée d'oublier ses limites. C'est ce qui fait de nos abattoirs des lieux évoquant trop souvent répulsion et dégoût.

\section{Don, Dieu, l'animal et la question du sujet}

Ces abattoirs - ainsi que tant d'autres manifestations de notre rapport industriel aux animaux — sont à la base des mouvements multiples qui luttent pour la réhabilitation de l'animal, y compris les tentatives pour leur donner une protection éthique et juridique et même un statut politique ${ }^{5}$. Cela a engendré (et engendre encore) une large discussion sur l'identité sociale et politique de l'animal. Qui est l'animal dans la société des hommes,

5. Sur la lutte pour les droits des animaux, voire par exemple Regan (2013). 
quelle est sa place, son identité ? Pour bien comprendre la portée de cette question, il n'est pas inutile de se demander de qui ou de quoi exactement on parle quand on parle de l'animal. Est-ce seulement de l'animal? En lui attribuant des droits, en lui donnant une place politique dans nos sociétés, est-ce exclusivement l'animal qu'on a en tête?

Ne parle-t-on pas autant de nous-mêmes, de notre position de sujet moderne étant séparé du monde sans avoir en nous-mêmes un fondement ontologique? La question de l'animal, la tentative de lui donner le statut de sujet, n'est-elle pas surtout motivée par le désir de trouver un certain support conférant un fondement plus stable au sujet humain?

Pour remédier à son problème le plus fondamental — la fissure qui annule son lien «essentiel» au monde et qui change ce monde en un univers d'objets "neutres» - l'homme d'aujourd'hui cherche dans un de ses "objets ", l'animal, le support pour résoudre ce problème. L'animal, lui aussi, est supposé être sujet. Car si l'animal était «sujet» comme lui, alors le sujet qu'est l'homme ne serait plus radicalement séparé du monde — et, par conséquent, ne serait plus fondé dans le vide d'une fissure, d'un «manque-à-être».

Est-ce qu'une telle opération résoudrait le problème? Est-ce que l'animal est capable de figurer dans une solution remédiant au statut précaire du sujet moderne? Ici, plutôt qu'une solution, la référence à l'animal fournit peut-être une manière de s'aveugler devant le problème. N'est-ce pas une stratégie de ne pas voir que, dans la modernité, notre point de vue notre sujet - est irrémédiablement localisé hors du monde, séparé de ce que nous ne pouvons considérer que comme des «objets»? En donnant aux animaux le statut de sujet, on fait comme si la nature du sujet moderne — le «point» dans lequel nous nous installons dans notre rapport à la réalité - était sans problème, parce qu'elle est liée de manière «naturelle » au monde.

Il est certainement justifié de considérer les animaux comme des "sujets de la vie», comme le fait le grand défenseur des droits des animaux, Tom Regan (2003, 80-83; 2013, 479-480), mais ils ne seront jamais des sujets modernes comme nous le sommes, c'est-à-dire sujets d'une liberté qui les placent épistémologiquement hors du monde: sujet d'une séparation du monde duquel pourtant ils vivent. Si nous considérons que les animaux sont des sujets comme nous, ce n'est peut-être jamais sans vouloir éviter la confrontation avec le problème profond qui caractérise notre condition de sujet moderne. Car, qu'est-ce que ce sujet? Séparé du monde par un doute hyperbolique (Descartes), se prétendant libéré même de 
l'essence que l'on est, qui alors est celui qui se prétend être le sujet de la liberté ? Qui est ce sujet qui prétend être le sujet de l'être tout en étant radicalement coupé de l'être?

Avant la modernité, ce sujet était Dieu. Celui-ci était séparé de l'être, si ce n'est que parce qu'il l'avait créé. Et c'était pourquoi les hommes se référaient au monde et s'inter-reliaient entre eux, non pas à partir d'euxmêmes, mais à partir de Dieu, ou plus exactement, à partir du fait que Dieu avait donné tout ce qui est, et qu'il l'avait donné tout spécifiquement à nous qui, pour cette raison (voici un autre de ses dons), étions dans la capacité d'avoir une connaissance du monde. C'était la réalité en tant que telle qui nous donnait activement à penser, et la science (médiévale) n'était au fond rien d'autre que le rapportage élaboré de ce don - et par conséquent, et sans la moindre contradiction, en même temps la vénération de son auteur, c'est-à-dire Dieu.

C'est ce sujet qui, au début du XviII ${ }^{\mathrm{e}}$ siècle, après un siècle et demi de guerres de religion, se trouvait dans l'incapacité de remplir sa tâche. Dieu ne fonctionnait plus comme "point» indiscutable à partir duquel l'homme fondait sa relation au monde. C'est-à-dire que l'homme ne se savait plus «donné » dans un monde qui lui-même était supposé donné à l'homme. Il ne pouvait plus partir de cette confiance non-questionnée par rapport au monde - ou, plus précisément, à l'essence du monde. Dès ces temps-là, l'homme ne pouvait avoir confiance qu'en lui-même, sans que cette confiance ait une base «ontologique» dans l'être avec qui il était en rapport. C'est cela le sujet moderne.

Il est vrai que Descartes pensait encore ce sujet comme un "être ", au sens ontologique du terme, comme un être pensant (res cogitans) qui différait radicalement de l'être du monde hors de lui, l'être des choses (res extensa). La postérité de Descartes n'a pas accepté ce dualisme intenable et a résolu le problème en annulant le res cogitans. Le matérialisme du XVIII $^{\mathrm{e}}$ siècle déclarait l'être un et indivisible, c'est-à-dire exclusivement matériel. Le spirituel - autre nom pour le sujet et son domaine - était considéré comme un produit de la fantaisie humaine, comme un "ghost in the machine».

Revoilà la question: qu'est le sujet? Comment concevoir son être? Depuis, le sujet ne pouvait être qu'un sujet à la recherche de son être, un sujet instable, inquiétant et anxieux d'être un jour confronté trop fatalement à son manque-à-être. Si ce sujet moderne est tenté de donner à l'animal le statut de sujet, ce n'est jamais sans l'intention de résoudre ce problème-ci. L'animal est supposé l'aider à lui redonner une base ontolo- 
gique, un fondement dans l'être. Il est censé combler le fossé fondamental qui sépare l'homme moderne de l'être du monde.

C'est dans ce sens que, dans notre modernité, la référence à l'animal remplit une fonction similaire à celle qui renvoie à Dieu. Au Moyen Âge, Dieu était le principe ontologique qui donnait à l'homme son «sujet". C'est pourquoi, une fois l'homme devenu lui-même «sujet» et constatant le sans-fond de ce qu'il était devenu, Dieu devenait l'objet d'une certaine «nostalgie métaphysique». C'est de lui qu'on attendait la restauration du lien rompu entre l'homme et le monde (ou l'être). La fascination pour l'animal dans notre modernité contemporaine révèle un désir similaire. L'animal donne à l'homme moderne l'opportunité de reconnecter son sujet «sans fond» avec le monde extérieur des «objets », un monde qui, lui, est supposé être le seul à avoir un fond (ontologique ou non). Si l'animal peut être considéré comme "sujet ", le sujet humain peut se savoir fondé dans le monde «objectif», dans la nature dont le statut ontologique est en tout cas moins incertain.

Ce «savoir» ne peut être que fantasmatique, car notre relation à la nature - en tant que réalisée dans nos sciences dites naturelles - ne peut que supposer un sujet "neutre", déconnecté précisément de cette nature qui est - et reste - son objet. Ce que les sciences naturelles ne peuvent penser, c'est le fait (pourtant très clair et difficile à dénier) que les données, les data, rassemblées et étudiées par les sciences, sont précisément données - que l'homme connait le monde parce que le monde lui est donné, tout comme il est lui-même donné au monde. C'est ce don qui joue aussi dans l'arrière-plan de notre fascination pour l'animal et la nouvelle place qu'on veut lui attribuer.

Si la théologie veut contribuer à la «question de l'animal» qui préoccupe nos contemporains, elle ne peut omettre d'y introduire le thème du don - le don comme paradigme d'un «être-au-monde " prémoderne persistant dans la modernité jusqu'à maintenant. De manière généralement inaperçue, le thème du don dirige «la question de l'animal ». Grâce à sa longue et multiple tradition, la théologie est une experte dans le paradigme du don, en tout cas du don monothéiste. Avec l'assistance des études anthropologiques sur ce même thème, la théologie est excellemment placée pour établir le cadre plus large dans lequel la portée profonde de la question de l'animal peut être éclaircie. Elle peut ainsi révéler la question qui est refoulée par nos tentatives de réhabiliter l'animal. Non pas que cette réhabilitation serait fausse, loin de là, mais elle n'est jamais sans l'intention 
de «résoudre» la question du sujet moderne, ce qui veut dire ici: nier sa condition foncièrement problématique.

Formulons la même chose dans un registre différent de celui du don. En attribuant à l'animal le statut de sujet égal à nous-mêmes, on manquerait l'opportunité de le voir comme autre, comme porteur d'une altérité qui pourrait contribuer à la déconstruction de notre égalité, de notre identité à nous-mêmes. S'il y a une égalité qui lie l'homme à l'animal, c'est une égalité dans la différence: c'est la différence avec l'animal qui confronte l'homme - ou plus exactement le «sujet » qu'il est — avec sa propre identité, une différence qui en est le fond, le «sujet». Tout comme, dans notre modernité avancée, Dieu est plus que jamais l'autre de l'homme. Mais c'est précisément dans son altérité que ce Dieu peut fonctionner comme un élément éminent dans le geste "déconstructeur " qui nécessairement accompagne le sujet moderne, trop sûr de lui-même et refoulant le "sans fond" sur lequel il se construit.

C’est dans l'altérité - dans la différence irréductible - que Dieu et l'animal se rencontrent. Pour le sujet moderne, l'animal et Dieu fonctionnent tous deux d'une manière semblable. Ils représentent ce que Giorgio Agamben (2006) a appelé «l'ouvert». Ou, pour le formuler dans l'idiome heideggérien, le Dasein — l'être-là, qui est l'homme — est défini comme une ouverture, une ouverture dans l'être qui donne à cet être l'opportunité d'avoir lieu, d'être là. Ainsi, l'animal, étant l'autre de l'homme, pour l'homme montre cet ouverture. C'est dans cette ouverture que l'homme peut redécouvrir le don qui lui «donne» la vie, y compris son identité. Quoiqu'impossible à réintroduire dans la modernité, le paradigme du don (de la manière dont il dirigeait les religions et le monothéisme) est essentiel pour soutenir l'esprit critique dans la modernité — une qualité d'esprit qui est indispensable à cette modernité. C'est une mission des religions monothéistes dans notre monde moderne, une mission qui, dans leurs doctrines, est loin d'être marginale, et qui s'avère même tout à fait centrale. La référence au Dieu monothéiste aussi bien qu'à l'animal est un élément indispensable pour un esprit critique, lui-même indispensable pour le sujet moderne.

\section{Références}

Agamben, G. (2006), L'ouvert: de l'homme et de l'animal, traduit de l'italien par Joël Garaud, Paris, Rivages Poches.

Assmann, J. (2007), Le prix du monothéisme, traduit par Laure Bernardi, Paris, Aubier. 
Balibar, E., Cassin, B. (2004), et al., "Sujet», dans B. Cassin, dir., Vocabulaire européen des philosophies: dictionnaire des intraduisibles, Paris, Le Robert / Seuil, p. 1233-1254.

Bichat, F.-X., (2013 [1800]), Recherches physiologiques sur la vie et la mort, Paris, Hachette.

Cassin, B. (réd.) (2004), Vocabulaire Européen des Philosophies - Dictionnaire des intraduisibles, Paris, Seuil / Le Robert.

Clarke, D. (2006), Descartes, A Biography, Cambridge, Cambridge University Press.

Descartes, R. (1901), CEuvres, tome IV: correspondance juillet 1643 à avril 1647, édition Charles Adam \& Paul Tannery, Paris, Cerf.

(1969), présentation et annotation par François Mizdrachi, Discours de la méthode, Paris, Union général d'éditions.

Drucker, P. (1965), Cultures of the North Pacific, Scranten (Pa), Chandler Publishing.

Fontenay, E. de (1998), Le silence des bêtes: la philosophie à l'épreuve de l'animalité, Paris, Fayard.

Hénaff, M. (2002), Le prix de la vérité: le don, l'argent, la philosophie, Paris, Seuil

Hamayon, R. (1995), "Pourquoi les jeux plaisent aux esprits ", dans G. ThinÈs et L. Heusch, dir., Rites et ritualisation, Paris, Vrin, p. 65-100.

Houston, W. (1993), Purity and Monotheism: Clean and Unclean Animals in Biblical Law, Sheffield, JSOT Press (Journal for the Study of the Old Testament, Supplement Series 140).

Hyde, L. (2007 [1979]), The Gift, New York, Vintage Books.

La MetTrie, Julien Offray de (1981), L'homme machine, Paris, Denoël / Gontier.

Mauss, M (2012), Essai sur le don - Formes et raison d'échange dans les sociétés archaïques, présentation de Florence Weber, Paris, Presses Universitaires de France (Quadrige) [article d'abord paru dans l'Année sociologique, seconde série, 1923-1924].

Milliet, J. (1987), Un allaitement insolite, dans J. Hainard et R. Kaehr, dir., Des animaux et des hommes, Neuchâtel, Musée d'ethnographie, p. 87-118. 
Milliet, J. (2003), «Entre douceur et violence: Le statut particulier des animaux allaités au sein par les femmes ", dans D. Blin, E. Thoueille et M. Soulé, dir., L'allaitement maternel: un dynamique à bien comprendre, Paris, Eres, p. 151-176.

Regan, Tom (2003), Animal Rights, Human Wrongs. An Introduction to Moral Philosophy, Lanham / Boulder / New York / Toronto / Oxford: Rowman \& Littlefield Publishers.

(2013), Les droits des animaux, traduit de l'américain par Enrique Utria, Paris: Harmattan.

Tавот, C. (2008), Le symbolique et le sacré - Théories de la religion, Paris, La Découverte.

Vernant, J.-P. et Detienne, M. (1972), La cuisine du sacrifice en pays grec, avec les contributions de J.-L. Durand, S. Georgoudi, F. Hartog et J. Svenbro, Paris, Gallimard.

\section{Résumé}

Les tentatives contemporaines de donner une place sociale et politique à l'animal et de lui attribuer le statut de «sujet » renvoie toujours aussi à la question concernant le sujet humain, plus spécifiquement le sujet de la modernité. C'est parce que nous sommes trop sûrs (c'est-à-dire pas du tout sûrs) concernant ce qu'est le «sujet moderne", que nous inventons la question sur l'animalité. D'une certaine manière, pour le problème du sujet de la modernité, la question de l'animal a une fonction similaire à la question de Dieu. C'est pourquoi une référence à la tradition théologique chrétienne est nécessaire pour comprendre l'enjeu de notre fascination actuelle pour l'animalité. Analysé à partir du paradigme du don (conçu par Marcel Mauss), l'essai offre une courte généalogie de la relation de l'homme à l'animal et montre comment la religion (dans toutes ses formes) y est profondément impliqué.

\section{Summary}

Today's attempts to give the animal a social and political place and to honor it with the status of "subject" refers to the question of the human subject and more precisely the subject of modernity. It is because we are too sure (which is to say not sure at all) concerning what the 'modern subject' is, that we invented the question of the animal. In a way, with regard to the pro- 
blem of the subject of modernity, the question of the animal fulfills a similar function as the question of God. This is why a reference to the Christian theological tradition is indispensable to understand what is at stake in the contemporary fascination for the animal. Analyzed from the perspective of the paradigm of the gift (as developed by Marcel Mauss), the essay offers a short genealogy of the human relation with regard to the animal and shows how religion (in all its shapes) is profoundly involved in it. 